\title{
Modeling Distance and Bandwidth Dependency of TOA-Based UWB Ranging Error for Positioning
}

\author{
Giovanni Bellusci, ${ }^{1}$ Gerard J. M. Janssen, ${ }^{1}$ Junlin Yan, ${ }^{2}$ and Christian C. J. M. Tiberius ${ }^{2}$ \\ ${ }^{1}$ Wireless and Mobile Communications Group (WMC), Delft University of Technology, 2628 CD Delft, The Netherlands \\ ${ }^{2}$ Mathematical Geodesy and Positioning Group (MGP), Delft University of Technology, 2629 HS Delft, The Netherlands
}

Correspondence should be addressed to Giovanni Bellusci, g.bellusci@tudelft.nl

Received 18 November 2008; Accepted 19 January 2009

Recommended by Luca De Nardis

A statistical model for the range error provided by TOA estimation using UWB signals is given, based on UWB channel measurements between 3.1 and $10.6 \mathrm{GHz}$. The range error has been modeled as a Gaussian random variable for LOS and as a combination of a Gaussian and an exponential random variable for NLOS. The distance and bandwidth dependency of both the mean and the standard deviation of the range error has been analyzed, and insight is given in the different phenomena which affect the estimation accuracy. A possible application of the model to weighted least squares positioning is finally investigated. Noticeable improvements compared to the traditional least squares method have been obtained.

Copyright ( $) 2009$ Giovanni Bellusci et al. This is an open access article distributed under the Creative Commons Attribution License, which permits unrestricted use, distribution, and reproduction in any medium, provided the original work is properly cited.

\section{Introduction}

Time of arrival (TOA) estimation using ultra-wideband (UWB) signals appears the most suitable ranging technique for indoor positioning applications which require centimeter- to decimeter-level accuracy [1]. For this reason, it is fundamental to provide an in depth investigation and modeling of the accuracy which can be achieved using this approach and its dependency on various system parameters. Some papers investigated and sometimes modeled the ranging performance achieved using UWB [2-6]; however, to the best of our knowledge, the related work reported in literature still lacks a complete and coherent understanding of the various factors which affect the range estimation accuracy, and of good statistical error models.

The contribution of this letter is to provide a better understanding of the types of range error usually experienced in indoor environments and to propose a novel statistical model for the error obtained by TOA-based UWB range estimation. Unlike in the available literature, the distance and the bandwidth dependency of both the bias of the range error, and its random variations have been investigated and statistically modeled. Finally, a possible application of the model to weighted least squares positioning is analyzed and the improvements compared to the classical least squares approach are evaluated.

\section{Measurement Setup, Signal Processing, and Range Estimation}

The channel impulse response (CIR) measurements used in this paper were collected using a time domain technique and cover the bandwidth between 3.1 and $10.6 \mathrm{GHz}$ allowed by the FCC for UWB radio transmissions. Details of the system setup can be found in [7]. The transmitter-receiver separation varied between 2 and $15 \mathrm{~m}$ for LOS, and between 3 and $12 \mathrm{~m}$ for NLOS. In NLOS, there is a physical obstruction, represented by walls, between the transmitter and the receiver. To allow distance dependency modeling, a total of about 600 measurements, each corresponding to different transmitter and receiver positions, were collected.

The CIR $h(t)$ is estimated by deconvolving the received signal in the frequency domain using the inverse filtering technique: the spectrum of the received signal is divided by the one of the reference signal, measured at a distance of one meter, in the absence of reflections. To model the bandwidth dependency of the range error, the spectra of the received 
signal and of the reference one are filtered with a rectangular window centered at $f_{c}=(3.1+10.6) / 2 \mathrm{GHz}=6.85 \mathrm{GHz}$, and with a bandwidth $B$ which varies from $0.5 \mathrm{GHz}$ to $7.5 \mathrm{GHz}$, increased in steps of $0.5 \mathrm{GHz}$. The first path of the CIR is defined as the first local maximum of the envelope of the estimated channel, with amplitude within $20 \mathrm{~dB}$ from the strongest peak. The range error is defined as $\epsilon=\hat{d}-d$, where $\hat{d}$ is the distance estimated by multiplying the TOA of the first path by the speed of light in air, and $d$ is the true distance between the transmitter and receiver.

\section{Range Error Results, and Modeling}

The range error is expressed as the sum of a bias $m$, plus a zero-mean random variable $S$ which accounts for the random variations around $m$. The proposed model explicitly considers the distance and bandwidth dependency of these two quantities:

$$
\epsilon(B, d)=m(B, d)+S(B, d) .
$$

In LOS, the bias $m(B, d)$ is due to multipath and reflects the fact that nonresolvable paths due to reflections always arrive later than the direct one. In NLOS, $m(B, d)$ is due to both multipath, as for LOS, and to the additional delay introduced by the propagation through dielectric materials. By analyzing the data, $m(B, d)$ has been modeled as a linearly increasing function of $d$; the slope of the linear fit increases with decreasing $B$ :

$$
m(B, d)=m_{B}(B) d+m_{0},
$$

where $m_{B}(B)$ accounts for its bandwidth dependency and it has been modeled as $m_{B}(B)=m_{1} \exp \left(-B / m_{2}\right)+m_{3}$. The coefficients $m_{0}, m_{1}, m_{2}$, and $m_{3}$ have been obtained by least squares fitting of the data corresponding to different bandwidths, and are reported in Table 1. $m(B, d)$ becomes larger for smaller $B$, since in this case, the number of nonresolvable multipath components that follow the direct path increases, resulting in more bias. At the same time, the bandwidth dependency becomes more evident with larger $d$. In fact in LOS, for increasing $d$, the direct path tends to have less weight with respect to the total signal, since the path loss exponent corresponding to the first path power is close to 2 [8], while that corresponding to the total power is usually between 1 and 2 [8], thus smaller; this means that multipath is more deteriorating for the resolvability of the direct path for larger $d$. Moreover, by increasing $d$, the distance difference between the direct path and the reflected ones tends to decrease, making the nonresolvable multipath more dense. For NLOS, the distance and bandwidth dependency of $m(B, d)$ can be explained with similar arguments; its significantly larger values compared to LOS, even for larger $B$, are due to the additional delay introduced by the propagation through dielectric materials.

The deviation from the mean, $S(B, d)$, has been modeled as a random variable with standard deviation $\sigma_{S}(B, d)$ which depends on both $B$ and $d$. As for $m(B, d)$, also $\sigma_{S}(B, d)$ becomes larger by decreasing $B$; in addition, to reflect the
TABLE 1: Parameters for the characterization of $m(B, d)$ and of $S(B, d)$.

\begin{tabular}{lcccc}
\hline$m(B, d):$ & $m_{0}(\mathrm{~m})$ & $m_{1}$ & $m_{2}(\mathrm{GHz})$ & $m_{3}$ \\
\hline LOS & 0 & 0.0148 & 0.48 & 0 \\
NLOS & 0.019 & 0.027 & 0.47 & 0.013 \\
\hline$S(B, d):$ & $\sigma_{S_{0}}(\mathrm{~m})$ & $\alpha$ & $g_{1}\left(\mathrm{~m}^{-\alpha}\right)$ & $g_{2}(\mathrm{GHz})$ \\
\hline LOS & 0.016 & 1.5 & 0.64 & 0.60 \\
NLOS & 0.049 & 1.5 & 0.21 & 0.73 \\
\hline
\end{tabular}

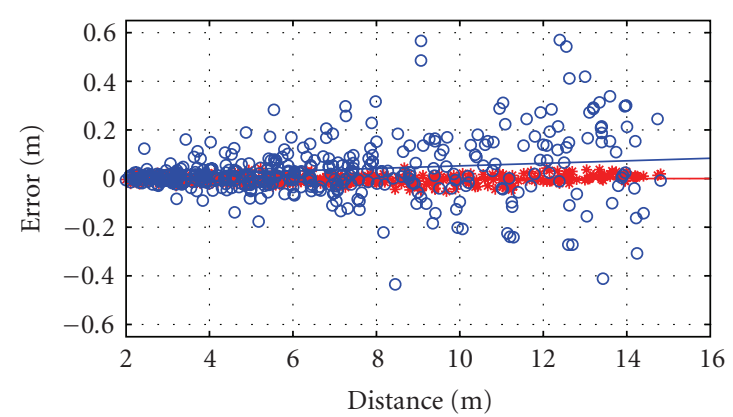

(a) LOS

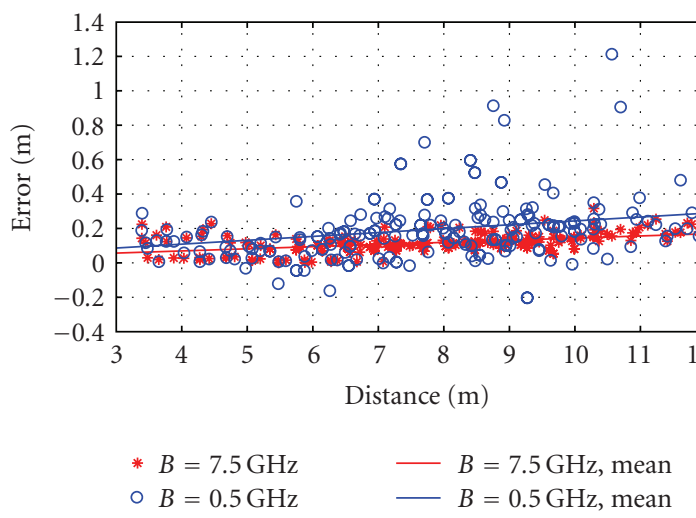

(b) NLOS

FIGURE 1: Range error versus $d$ for (a) LOS and for (b) NLOS, for $B=7.5 \mathrm{GHz}$ and $B=0.5 \mathrm{GHz}$.

fact that for the full bandwidth no distance dependency of $\sigma_{S}(B, d)$ has been observed, while for smaller bandwidths, $\sigma_{S}(B, d)$ significantly increases with $d$, the following model for $\sigma_{S}(B, d)$ is proposed:

$$
\sigma_{S}(B, d)=\sigma_{S_{0}}\left(g_{B}(B) g_{d}(d)+1\right)
$$

with $g_{B}(B)=g_{1} \exp \left(-B / g_{2}\right)$ and $g_{d}(d)=d^{\alpha}$. This behavior for $\sigma_{S}(B, d)$ can be justified observing that due to multipath, the resolvability of the direct path becomes worse for smaller bandwidths or larger distances, as previously motivated in the analysis of $m(B, d)$. The parameters $\sigma_{S_{0}}, \alpha, g_{1}$, and $g_{2}$ have been obtained by least squares fitting of the data; their values are also reported in Table 1. Figure 1 presents the scatter plot of the range error versus distance both for (a) LOS and for (b) NLOS, for $B=7.5$ and $B=0.5 \mathrm{GHz}$ for comparison. 
For the statistical characterization of $S(B, d)$, a difference between the two propagation conditions arises. Therefore, we introduce the new random variable

$$
S_{0}=\frac{S(B, d)}{g_{B}(B) g_{d}(d)+1}
$$

which represents $S(B, d)$ normalized to the terms which model its bandwidth and distance dependency. By analyzing the histogram of $S_{0}$, for LOS, it has been modeled as a zero mean Gaussian random variable with standard deviation $\sigma_{S_{0}}$. For NLOS, we noticed an asymmetric distribution around the zero mean, for all bandwidths. In fact, the tail corresponding to the positive values of the histogram of $S_{0}$ is longer than that corresponding to the negative ones. The physical explanation of this aspect is the following: in NLOS, with strong multipath conditions, there are situations in which several reflected and nonresolvable paths may arrive closely after the direct one; this can move the detected first path of the CIR to a delay which is significantly larger than $1 / B$. On the contrary, the negative errors due to multipath are always limited to approximately $1 / B$. Figure 2 explains this point. This phenomenon was not experienced in LOS due to the less dense multipath. To account for this aspect, in NLOS, $S_{0}$ has been modeled as the sum of two independent random variables

$$
S_{0}=s_{N} R_{N}+s_{E} R_{E}+s_{0},
$$

where $R_{N}$ is a standard Gaussian random variable and $R_{E}$ is a standard exponential random variable. The coefficients $s_{N}=$ $w \sigma_{S_{0}}, s_{E}=\sqrt{1-w^{2}} \sigma_{S_{0}}$, and $s_{0}=-s_{E}=-\sqrt{1-w^{2}} \sigma_{S_{0}}$ can be expressed as functions of only one variable $w(0 \leq w \leq 1)$, and are necessary to weight the two distributions with the constraints for the mean and the standard deviation of $S_{0}$. By fitting the data, we obtained $w=0.6$.

From the described model, it is possible to derive the mean and standard deviation of the range error obtained from the total set of measurements, as a function of $B$. From (1) and (2), the global mean range error results in

$$
\operatorname{mean}\{\epsilon(B, d)\}=m_{B}(B) E[d]+m_{0},
$$

where $E[\cdot]$ represents the expectation operation. $E[d]=$ $6.95 \mathrm{~m}$ for LOS and $E[d]=7.74 \mathrm{~m}$ for NLOS has been evaluated from the measured data. For the standard deviation of the range error, using (1)-(4), $\epsilon(B, d)$ can be expressed as

$$
\epsilon(B, d)=m_{B}(B) d+m_{0}+S_{0}\left(g_{B}(B) d^{\alpha}+1\right),
$$

which is a nonlinear function of two independent random variables, $d$ and $S_{0}$. Using the variance propagation law applied to (7), linearized around the means of $d$ and $S_{0}$, and approximated up to its second order, we find

$$
\operatorname{std}\{\epsilon(B, d)\} \cong \sqrt{\left(m_{B}(B) \sigma_{d}\right)^{2}+\left(\sigma_{S_{0}}\left(g_{B}(B)(E[d])^{\alpha}+1\right)\right)^{2}},
$$

where $\sigma_{d}=3.41 \mathrm{~m}$ for LOS, and $\sigma_{d}=2.05 \mathrm{~m}$ for NLOS is the standard deviation of the distribution of the distances, which has been evaluated from the measured data.

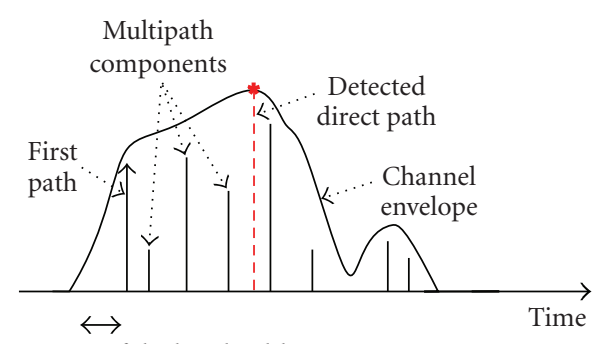

Inverse of the bandwidth

FIGURE 2: NLOS range error behavior.

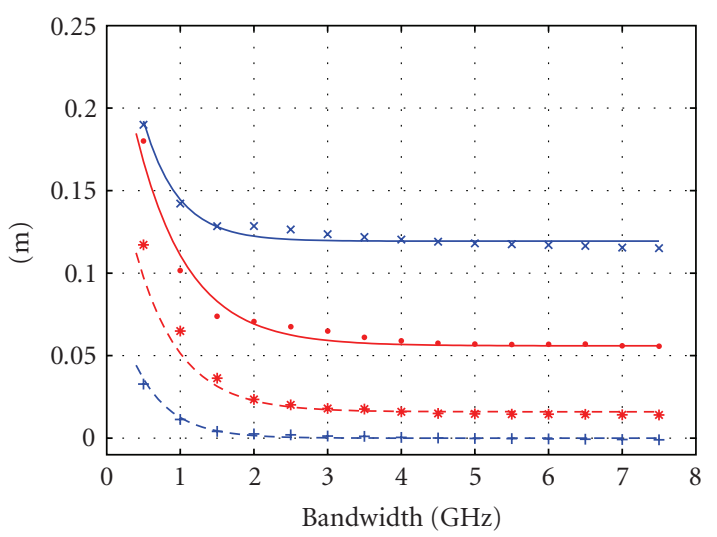

$$
\begin{aligned}
& \text { Mean range error Std range error } \\
& \times \text { NLOS, data - - LOS, model } \\
& \text { — NLOS, model } \quad * \quad \text { LOS, data } \\
& + \text { LOS, data - NLOS, data } \\
& \text { - - - NLOS, model _ _ NLOS, model }
\end{aligned}
$$

FIgURE 3: Mean and standard deviation of the global range error versus $B$.

Figure 3 shows the mean and the standard deviation of the range error directly obtained using the total set of data, and the model proposed in (6)-(8) for comparison.

\section{Weighted Least Squares Positioning}

In this section, a possible application of the proposed model to improve the classical least squares positioning is investigated. From the total set of measurements, a subset of $n$ of them is randomly chosen. A pair $(\theta, \phi)$ is associated to each of the $n$ selected ranges. In this way, a reference point with spherical coordinates $(d, \theta, \phi)$ in the three-dimensional space is associated to each measurement, and a simulated positioning system is obtained from the $n$ selected ranges. $\theta$ is the angle between the $z$-axis and the reference point, $\phi$ is the angle between the $x$-axis and the projection of the reference point on the $x-y$ plane. The axis origin represents the unknown position to be estimated using the set of the $n$ corresponding range estimations $\hat{d}$. The general system model can be written as $\underline{d}=A\left(\underline{x}_{u}\right)+\underline{\epsilon}$, where the underline indicates vector quantities. $A(\cdot)$ is a mapping from $R^{3}$ to $R^{n}$, $\underline{d}$ and $\underline{\epsilon} \in R^{n}$, and $\underline{x}_{u}=(0,0,0)$. From the vector of the estimated distances, an estimate $\underline{\hat{x}}_{u}$ of $\underline{x}_{u}$ can be obtained. 
TABLE 2: Final position estimation accuracy for different scenarios.

\begin{tabular}{lcccccc}
\hline & LOS & LOS & NLOS & NLOS & LOS/NLOS & LOS/NLOS \\
$n=4$ & $n=8$ & $n=4$ & $n=8$ & $n$ \\
\hline$B=7.5 \mathrm{GHz}$ & & & & & \\
LS & $0.025(\mathrm{~m})$ & $0.017(\mathrm{~m})$ & $0.143(\mathrm{~m})$ & $0.093(\mathrm{~m})$ & $0.167(\mathrm{~m})$ & $0.064(\mathrm{~m})$ \\
WLS & $0.025(\mathrm{~m})$ & $0.017(\mathrm{~m})$ & $0.091(\mathrm{~m})$ & $0.068(\mathrm{~m})$ & $0.054(\mathrm{~m})$ & $0.024(\mathrm{~m})$ \\
\hline$B=0.5 \mathrm{GHz}$ & & & & & \\
LS & $0.211(\mathrm{~m})$ & $0.147(\mathrm{~m})$ & $0.322(\mathrm{~m})$ & $0.210(\mathrm{~m})$ & $0.308(\mathrm{~m})$ & $0.180(\mathrm{~m})$ \\
WLS & $0.180(\mathrm{~m})$ & $0.102(\mathrm{~m})$ & $0.235(\mathrm{~m})$ & $0.160(\mathrm{~m})$ & $0.207(\mathrm{~m})$ & $0.121(\mathrm{~m})$ \\
\hline
\end{tabular}

The performance of the positioning algorithms is evaluated by calculating $\epsilon_{\underline{\hat{x}}_{u}}=E\left[\left\|\underline{\hat{x}}_{u}\right\|\right]$, which represents the root mean square error in the position estimation.

In traditional least squares, the problem is solved by searching the minimum of the objective function

$$
\underline{\underline{x}}_{u}^{\mathrm{LS}}=\arg \min _{\underline{x}_{u}}\left\|\underline{d}-A\left(\underline{x}_{u}\right)\right\|^{2} .
$$

If the error model is known, a weighted and unbiased estimation of the position can be obtained

$$
\underline{\hat{x}}_{u}^{\mathrm{WLS}}=\arg \min _{\underline{x}_{u}}\left\|\underline{d}-\underline{m}(B, d)-A\left(\underline{x}_{u}\right)\right\|_{Q_{d d}^{-1}}^{2}
$$

where $Q_{d d}$ is the variance matrix (a diagonal matrix containing as the $[j, j]$ element the variance of the $j$ th measured distance). Since both $m(B, d)$ and $Q_{d d}$ depend on the real distance which is unknown, the estimated distance, instead of the unknown real distance, has been used for their evaluation in (10). Six different scenarios have been simulated: LOS and $n=4$, LOS and $n=8$, NLOS and $n=4$, NLOS and $n=8, n$ $=4$ with 2 measurements in LOS and 2 in NLOS, and $n=8$ with 4 measurements in LOS, and 4 in NLOS. Table 2 shows the obtained results, averaged over 10000 repetitions, for the two methods proposed in (9), (10), and for $B=0.5 \mathrm{GHz}$ and $B=7.5 \mathrm{GHz}$, for comparison. Only for the case of LOS and $B=7.5 \mathrm{GHz}$, there is no improvement using the range error model knowledge, since the range error in this case is unbiased and it does not depend on the distance. For all the other scenarios, the range error model knowledge always allows to improve the final position estimation accuracy up to about $300 \%$ when combining together LOS and NLOS measurements for $B=7.5 \mathrm{GHz}$.

\section{Conclusions}

In this letter, a statistical model for the range error obtained by TOA estimation using UWB signals has been proposed. It is shown that both a decrease of the bandwidth and an increase of the distance cause an increase in the mean and standard deviation of the range error due to the more dense multipath which results in these situations. The range error is modeled as a Gaussian random variable for LOS, and as the combination of a Gaussian and an exponential random variable for NLOS. The more complex characterization in NLOS describes the effects on the range error of the dense multipath in this propagation condition, and represents a generalization which includes also the LOS scenario. A possible application of the model to weighted least squares positioning is finally investigated. Improvements compared to traditional least squares are especially evident when there is a significant redundancy or a combination of LOS and NLOS measurements.

\section{References}

[1] S. Gezici, Z. Tian, G. B. Giannakis, et al., "Localization via ultrawideband radios: a look at positioning aspects of future sensor networks," IEEE Signal Processing Magazine, vol. 22, no. 4, pp. 70-84, 2005.

[2] Z. Irahhauten, G. Bellusci, G. J. M. Janssen, H. Nikookar, and C. C. J. M. Tiberius, "Investigation of UWB ranging in dense indoor multipath environments," in Proceedings of the 10th IEEE Singapore International Conference on Communication Systems (ICCS '06), pp. 1-5, Singapore, October 2006.

[3] C.-C. Chong, F. Watanabe, and M. Z. Win, "Effect of bandwidth on UWB ranging error," in Proceedings of IEEE Wireless Communications and Networking Conference (WCNC '07), pp. 1559-1564, Kowloon, China, March 2007.

[4] C. Gentile and A. Kik, "WLC28-4: an evaluation of ultra wideband technology for indoor ranging," in Proceedings of IEEE Global Telecommunications Conference (GLOBECOM '06), pp. 1-6, San Francisco, Calif, USA, November-December 2006.

[5] B. Alavi and K. Pahlavan, "Modeling of the distance error for indoor geolocation," in Proceedings of IEEE Wireless Communications and Networking (WCNC '03), vol. 1, pp. 668-672, New Orleans, La, USA, March 2003.

[6] B. Alavi and K. Pahlavan, "Modeling of the TOA-based distance measurement error using UWB indoor radio measurements," IEEE Communications Letters, vol. 10, no. 4, pp. 275-277, 2006.

[7] Z. Irahhauten, A. Yarovoy, H. Nikookar, G. J. M. Janssen, and L. Ligthart, "Measurement setup for ultra wide band indoor radio channel characterization," in Proceedings 11th Symposium on Communications and Vehicular Technology in the Benelux (SCVT '04), Gent, Belgium, November 2004.

[8] G. Bellusci, G. J. M. Janssen, J. Yan, and C. C. J. M. Tiberius, "Low complexity ultrawideband ranging in indoor multipath environments," in Proceedings of IEEE/ION Position, Location and Navigation Symposium (PLANS '08), pp. 394-401, Monterey, Calif, USA, May 2008. 

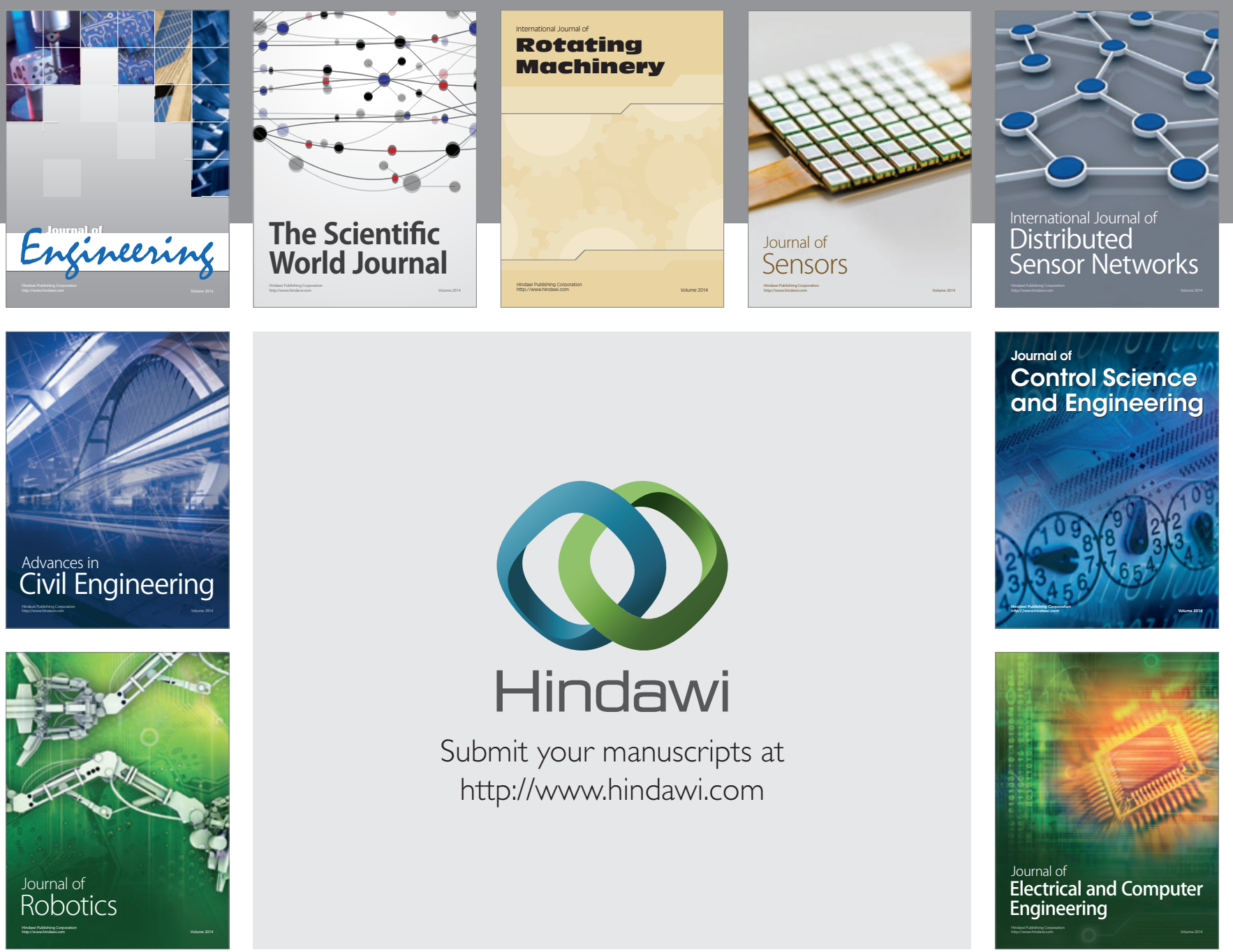

Submit your manuscripts at

http://www.hindawi.com
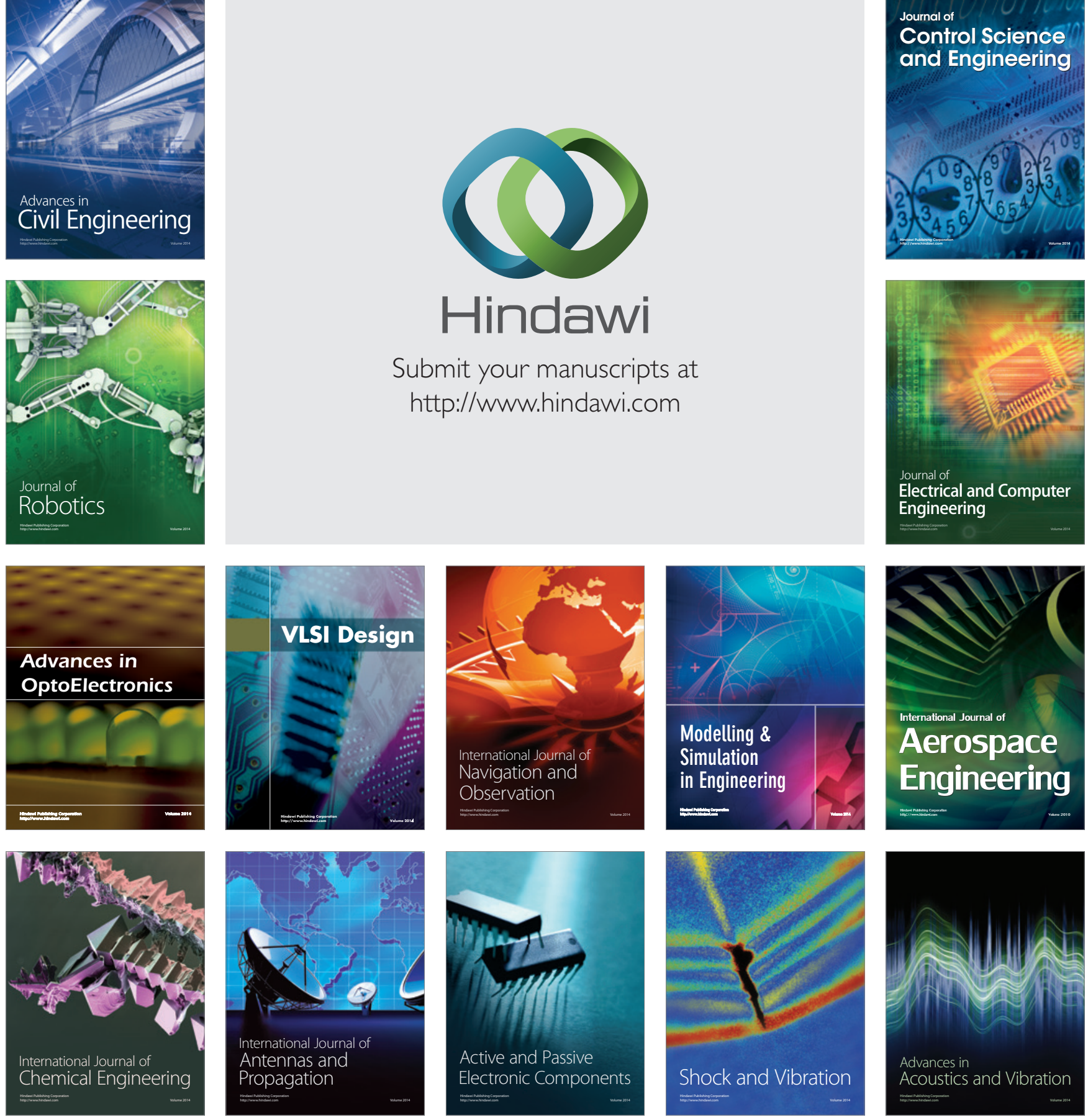